\title{
PARÁMETROS DE DISEÑO ORGANIZACIONAL: INCIDENCIA EN LA PRODUCTIVIDAD Y EN LOS VÍNCULOS SOCIALES*
}

\author{
DAIMER HIGUITA LÓPEZ" \\ UNIVERSIDAD NACIONAL DE COLOMBIA
}

Recibido/ Received/Recebido: 17/01/2011 - Aceptado/ Accepted/Aprovado: 14/03/2011

\begin{abstract}
Resumen
Este artículo presenta ciertos efectos que algunos parámetros del diseño organizacional tienen sobre los vínculos sociales entre los trabajadores, impactando la cooperación y la productividad en la empresa. La investigación, realizada con un enfoque cualitativo, se basó en observaciones y entrevistas semi-estructuradas. De este modo, a partir de los referentes teóricos tratados, se configuraron las categorías de análisis que permitieron interpretar los hallazgos del estudio. Como resultado se encontró que los aspectos del diseño organizacional que generan un costo emocional y social para los empleados debido a la ruptura de sus vínculos son: la departamentalización, el puesto de trabajo, la jerarquía, las reglas y normas.
\end{abstract}

Palabras clave: Diseño organizacional, empresas, puesto de trabajo, normas, departamentalización, productividad, vínculos sociales.

\section{ORGANIZATIONAL DESIGN PARAMETERS: INCIDENCE ON PRODUCTIVITY AND SOCIAL LINKAGES}

\begin{abstract}
This article presents the effects that some organizational design parameters have on social linkages among workers, impacting on enterprise cooperation and productivity. The research, made with a qualitative approach, was based on observations and semi-structured interviews. Thereby, from theoretical references, the analysis categories were designed which allowed the interpretation of the findings. As a result, it was found that organizational design aspects that generate an emotional and social cost to the employees due to linkages break are: departmentalization, workplace, hierarchy, rules and standards.
\end{abstract}

Keywords: Organizational design, enterprises, workplace, standards, departmentalization, productivity, social linkages.

El artículo se origina en los resultados de la investigación cualitativa titulada "La estructura formal de organizaciones productivas y su incidencia en la noción de cooperación espontánea", y desarrollada entre junio de 2006 y septiembre de 2007.

Administrador de Empresas (Universidad de Antioquia, 2000), Magister en Ciencias de la Administración (Universidad EAFIT, 2007), Profesor de la Escuela de Administración y Contaduría, Universidad Nacional de Colombia. Grupo de Investigación "Emprendimiento y Mipymes". Estudiante de doctorado en Ciencias Económicas. Correo electrónico: dhiguita@unal.edu.co 


\title{
PARAMÉTROS DE DESENHO ORGANIZACIONAL: INCIDÊNCIA NA PRODUCTIVIDADE E NOS VÍNCULOS SOCIAIS
}

\begin{abstract}
Resumo
Este artigo apresenta efeitos de alguns parâmetros do desenho organizacional sobre os laços sociais entre os trabalhadores, que afetam a cooperação e a produtividade na empresa. Esta pesquisa qualitativa foi baseada em observações e entrevistas semi-estruturadas. Assim, com base nos referentes teóricos, construíram-se as categorias de análise para interpretar os resultados do estudo. Como resultado verificou-se que os aspectos do desenho organizacional que criam um custo emocional e social aos funcionários, devido à ruptura de suas ligações, são a departamentalização, o local de trabalho, a hierarquia, as regras e os regulamentos.

Palavras chave: Desenho organizacional, firmas, sítio de trabalho, regras, departamentalização, produtividade, laços sociais
\end{abstract}

Higuita, D. (2011). Parámetros de diseño organizacional: Incidencia en la productividad y en los vínculos sociales. En: Revista de la Facultad de Ciencias Económicas de la Universidad Militar Nueva Granada. rev.fac.cienc.econ,XIX (1).

JEL: M10, M12, M19.

\section{Introducción}

Para Goodin (1996), el cambio en las organizaciones puede darse por azar, por evolución o por intervenciones deliberadas para propósitos específicos.

"Considerar que el cambio puede ser objeto de intervenciones intencionales pone en discusión dos aspectos: de una parte, que las organizaciones pueden ser objeto de diseño y en consecuencia que el futuro puede ser construido, y de otra parte, la manera cómo el propio marco institucional modela a los "diseñadores" (Barrera, Rodríguez y Bedoya, 2008, 159).

El diseño organizacional es un tema importante en el pensamiento y la práctica de la gestión. El diseño $y$ rediseño de las organizaciones es una actividad central de consultores y ha sido un punto clave de esta disciplina. En la literatura sobre el diseño organizacional, se han desarrollado tres perspectivas diferentes: la racional, la dialógica, y la perspectiva pragmática. Estos enfoques abarcan distintos puntos de vista y tienen ideas diferentes acerca de la elaboración de actividades de diseño (Visscher \& Voerman, 2010). Algunas decisiones administrativas, como el diseño organizacional, necesarias para la eficiencia de la organización, tienen igualmente efectos en las personas, actualmente denominadas colaboradores.

Este artículo busca develar el impacto de algunos parámetros del diseño organizacional en la productividad y en las relaciones sociales entre los trabajadores. En primer lugar, se mencionan sucintamente las perspectivas y la investigación en el tema, la necesidad de cooperación en la empresa y algunos elementos de la estructura formal. Luego se detalla la metodología empleada durante la investigación que originó el presente artículo y las de técnicas de recolección de información. Posteriormente se describe la manera como la jerarquía, el puesto de trabajo, las normas y la departamentalización funcional afectan la cooperación espontánea, la cual contribuye tanto con la productividad en la empresa como con la cohesión social. Y, finalmente, el texto presenta las conclusiones. 


\section{Perspectivas e investigación en diseño organizacional}

Si bien durante el texto se mencionan algunas conclusiones de investigaciones en el campo del diseño organizacional, en las siguientes líneas se hace una revisión sucinta de este tema, dado el interés del presente artículo. Pero antes de ello, veamos algunas diferencias entre las perspectivas pragmática, dialógica y racional en diseño. Dentro del enfoque racional, el diseño es visto como la construcción de modelos para las características estructurales de las organizaciones. Tradicionalmente, estas características estructurales se refieren a la estructura formal de la organización (Visscher \& Voerman, 2010, 715).

El enfoque de diseño dialógico es analítico en lugar de prescriptivo. Este enfoque es similar al racional en las características estructurales de la organización. Se diferencia en que las organizaciones son vistas como sistemas políticos, y se parte de que las estructuras sirven a los intereses de las personas que tienen el poder en la organización.

De otro lado, mientras que el enfoque racional se refiere a la complejidad como algo que debe reducirse, y el dialógico percibe la complejidad principalmente como producto de las diferencias políticas, el enfoque pragmático trata de absorber la complejidad presente en la organización (Visscher \& Voerman, 2010).

Tabla 1. Parámetros de diseño según Henry Mintzberg ${ }^{1}$

\begin{tabular}{|c|c|c|}
\hline Grupo & Parámetro de diseño & Conceptos relacionados \\
\hline Diseño de posiciones & $\begin{array}{l}\text { Especialización de tarea } \\
\text { Formalización de comportamiento } \\
\text { Capacitación y adoctrinamiento }\end{array}$ & $\begin{array}{l}\text { División básica de trabajo } \\
\text { Estandarización de contenido de trabajo }\end{array}$ \\
\hline Diseño de superestructura & $\begin{array}{l}\text { Agrupamiento de unidad } \\
\text { Dimensión de la unidad }\end{array}$ & $\begin{array}{l}\text { Supervisión directa } \\
\text { División administrativa del trabajo } \\
\text { Sistemas de autoridad formal } \\
\text { Organigrama }\end{array}$ \\
\hline Diseño de encadenamientos laterales & ------- & Sistema de flujos regulados \\
\hline Diseño de sistema de toma de decisiones & $\begin{array}{l}\text { Descentralización vertical } \\
\text { Descentralización horizontal }\end{array}$ & Sistemas de autoridad formal \\
\hline
\end{tabular}

1 Tomada de: Diseño de organizaciones eficientes. Henry Mintzberg (2001, 36).
Los estudios presentados en el simposio sobre diseño organizacional (realizado en California en 2009), establecen con claridad los vínculos entre los elementos básicos del diseño organizacional y muestran cómo las decisiones deben tomarse de manera concertada con tales elementos, así como con la visión que orienta el negocio (Beckman, 2009).

Por su parte los estudios de Morgeson muestran como los contextos laborales ejercen influencias significativas tanto en las características del puesto de trabajo como en los resultados individuales. Además, los diferentes cargos y ocupaciones tienen sus propias características culturales que pueden ser tan influyentes como los efectos normalmente atribuidos a las culturas organizacionales (Morgeson et al, 2010).

Cuando las características de un individuo están en línea con lo que el cargo ofrece en términos de los refuerzos, los resultados individuales se reflejan en mayores niveles de satisfacción en el trabajo. En los textos La estructuración de las organizaciones (1979) y Diseño de organizaciones eficientes (2001), Henry Mintzberg describe nueve parámetros de diseño organizacional, incluidos en cuatro grupos, como se muestra en la tabla № 1 . Uno de estos grupos es el diseño de puestos, que para Mintzberg comprende tres parámetros que son: especialización de la tarea, formalización del comportamiento y capacitación y adoctrinamiento. 


\subsection{Parámetros de diseño según Henry Mintzberg}

Para Beckman (2009), al momento de elaborar el diseño de una organización es necesario comprender qué opciones existen para el diseño y qué características de la organización se pueden cambiar. Adicionalmente, se requiere probar las opciones de diseño, antes de su implementación. Sin embargo, y como afirma Galbraith, los esfuerzos de diseño de la mayoría de las organizaciones se centran demasiado en la estructura, haciendo caso omiso de la importancia de los demás elementos del diseño (Beckman, 2009; Zapata, 2008).Después de estos breves comentarios sobre el diseño, se hará mención al concepto de cooperación y a los vínculos sociales, para abordar posteriormente la estructura formal en las organizaciones.

\subsection{La cooperación espontánea y los vínculos sociales en la organización}

Una organización puede entenderse como un conjunto de personas que colaboran conforme a unos lineamientos para alcanzar unas metas específicas (Hodge, Anthony \& Gales, 1998, 11). Para Barnard, la organización es un sistema cooperativo en el que todas las partes del sistema colaboran con un aspecto de la organización y contribuyen, entre todas, al logro de los objetivos institucionales, pues:

"la cooperación es un aspecto social de una situación particular" (Barnard, 1971, $60)$.

En este sentido, la cooperación implica algún tipo de interacción. Aunque existen distintos tipos de cooperación, la investigación se delimitó a analizar la cooperación espontánea ${ }^{2}$, la cual es una forma de cooperación:

"No prescrita por la tradición, el contrato o la autoridad; es de carácter situacional, y prácticamente constituye la esencia de las relaciones dentro de la familia, la comunidad, el equipo y otras formas asociativas personales e íntimas. (...) Independientemente de la clase de tareas asignadas, la cooperación espontánea es menos probable cuando no ha habido oportunidad de que surja una relación amistosa. (...) En el ejército, en las empresas, o en la administración, dadas las condiciones propicias, aparecen formas de cooperación espontánea que pueden favorecer (o invalidar) las de tipo planificado o dirigido." (Nisbet, 1974, 183).

Así entonces, todas aquellas prácticas de ayuda mutua entre las personas, sin que medie entre ellas ningún contrato, puede denominarse cooperación espontánea. Pero ¿por qué se generan esas prácticas espontáneas entre las personas? Porque éstas tienen propósitos comunes o afinidades en los modos de pensar, trabajar o vivir, lo cual las lleva a querer afianzar vínculos entre ellas. Y ¿por qué se crea esta comunión de intereses y vínculos? Posiblemente porque, producto de la interacción social, se presenta una cierta identificación entre los sujetos, entendida:

"Como la manifestación más temprana de un enlace afectivo a otra persona [y que] puede surgir siempre que el sujeto descubre en sí un rasgo común con otra persona" (Freud, 1981, 2587).

Ahora bien, la interacción fue objeto de estudio de Durkheim, quien acuñó los conceptos solidaridad mecánica y solidaridad orgánica, advirtiendo que en la solidaridad mecánica, los vínculos entre las personas de las sociedades primitivas se establecían espontáneamente, mientras que en la sociedad industrial, estos vínculos eran determinados racionalmente como consecuencia de la división del trabajo. Para Durkheim, en la solidaridad mecánica:

2 Nisbet (1974) traza una tipología según la cual la cooperación puede ser automática (donde existe comunidad de intereses directamente derivados de una posición ecológica), tradicional (no regida por el instinto ni por la voluntad, sino más bien por normas sociales de carácter tradicional), contractual (regida por normas legislativas, tanto en lo que se refiere a la duración de la cooperación, como a los requisitos concretos de la relación), dirigida (el resultado del reconocimiento común de un objetivo bien definido). 
"Domina a la sociedad un conjunto muy firme de sentimientos y creencias compartidos por todos los miembros de la comunidad" (Giddens, 1998, 142).

Mientras que la solidaridad orgánica está estableciendo lo social de manera muy diferente, y no precisamente desde lo común, pues ella:

\begin{abstract}
"No arranca simplemente de la aceptación de un conjunto de creencias y sentimientos comunes, sino de la interdependencia funcional en la división del trabajo" (Giddens, 1998, 142).
\end{abstract}

La interacción social contribuye con la construcción de vínculos entre las personas y, de esta forma, con la cohesión social de un grupo. En este sentido, esos vínculos facilitan eventos de solidaridad y cooperación entre los miembros del grupo. Por consiguiente, en los espacios colectivos que emergen por medio de la solidaridad mecánica expuesta por Durkheim, existen puntos de identificación entre las personas, que les permiten cooperar entre sí $^{3}$. En contraste, la solidaridad orgánica propone una cooperación no a partir de rasgos identificatorios o afinidades entre los sujetos, si no a partir de su funcionalidad o utilidad para la organización. Véase la Ilustración № 1 .

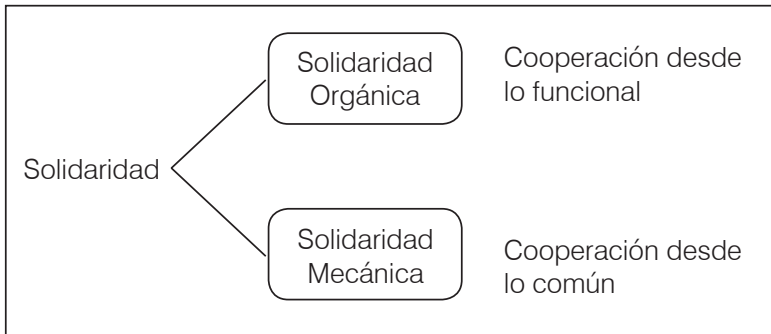

Ilustración 1. Características de la cooperación desde los vínculos sociales ${ }^{4}$

\section{Características de la cooperación desde los vínculos sociales}

Para el caso del campo organizacional, es posible que algunos empleados deseen cooperar entre sí, por el vínculo que han construido gracias a la posibilidad de compartir habilidades, comunicarse y de dar solución a las dificultades laborales (Véase la Ilustración 2). En las organizaciones contemporáneas se pueden observar vinculaciones fundamentadas en la solidaridad tanto mecánica como orgáni$c a$, que podrían asociarse con relaciones informales y formales entre los trabajadores. Dichas relaciones están fundamentadas en aspectos diferentes, como lo sugiere Aktouf, cuando señala que:

"Si la lógica que guía el funcionamiento de la empresa es económica y racional, la lógica de los miembros de la empresa pertenece a la afectividad y los sentimientos. El trabajo del administrador se ve de esta manera modificado, ya que debe tener en cuenta una organización informal que posee su propia dinámica y sus propios objetivos" (Aktouf, 2001, 249).

Por consiguiente, se hace importante rescatar el valor del trabajador como persona, pues en nuestro medio ha hecho carrera la idea de que las personas son costosas, y por lo tanto toda estrategia tiene por finalidad "reducir costos laborales", lo cual se ha convertido en condición para ser competitivos (Calderón, 2004, 65).

Pero dicha competitividad está atravesada por un eficiente trabajo en equipo. Y como demostraron Wilkens \& London (2006), los equipos de alto desempeño (basado en la calidad, la velocidad de la realización, y la cooperación entre miembros) se caracterizan por los altos grados de integración de sus miembros.

\footnotetext{
3 En términos generales, la identificación puede entenderse como un mecanismo psíquico consciente o inconsciente que induce a un sujeto a comportarse, pensar, vincularse y sentir como otro que actúa como su modelo o encuentra en él afinidad en su comportamiento y el propio (Ver Abbagnano, 1997).

4 Fuente: Construcción propia.
} 
De igual forma, Robbins (2004) sostiene que la comunicación y la cohesión social son esenciales para la cooperación y el desempeño eficiente de los grupos de trabajo. Pero dicha cohesión social y la cooperación espontánea solo podrán emerger a partir de los vínculos que se crean entre las personas, producto de la interacción social entre ellas.

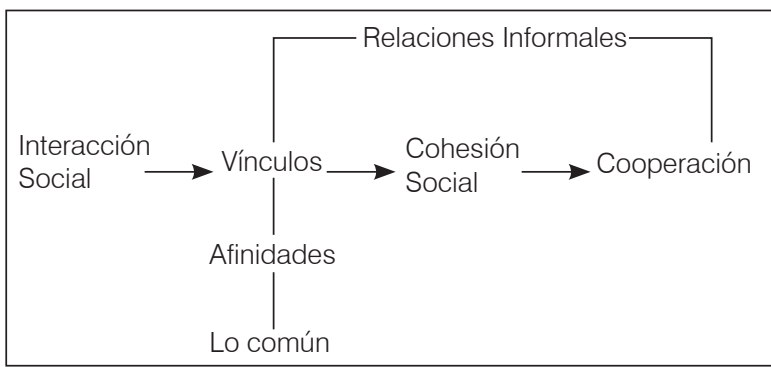

Ilustración 2. Relación entre la interacción y la cooperación ${ }^{5}$

\section{Elementos del diseño organizacional}

Es necesario precisar que cuando se habla de estructura formal no se hace referencia exclusivamente al ordenamiento organizacional que queda plasmado en el organigrama, sino que el concepto está apuntando a aquellos postulados que desde la prescripción se refieren a la forma como se relacionan (o se deben relacionar) los empleados para que contribuyan con los objetivos de la empresa. De esta manera lo afirma Jorge Etkin cuando señala que la estructura se:

"Refiere a la forma en que se divide y asigna el trabajo, y también a las relaciones a través de líneas de autoridad y redes de comunicación" (Etkin, 2000, 25).

El diseño organizacional es la manera como la estructura formal establece, con Ilustración y formaliza la posición, el funcionamiento y las líneas formales de relación entre los elementos que componen la organización ${ }^{6}$.
"El diseño supone el albedrío, una capacidad para alterar un sistema. En el caso de la estructura organizativa, diseño significa girar aquellas manijas que influyen sobre la división del trabajo y los mecanismos coordinadores" (Mintzberg, 2001, 33).

Afectando así la forma en que funciona la organización. El éxito del diseño requiere profunda comprensión del contexto para el cual la organización se está diseñando, el entorno en el que compite la empresa, las estrategias de negocio que va a utilizar, y las capacidades que necesita para competir (Beckman, 2009). A lo que podemos agregar el factor humano en interacción.

Los parámetros del diseño organizacional que surgieron como resultado de la investigación son departamentalización funcional, puesto de trabajo, jerarquía y reglas. Veamos algunas apreciaciones sobre éstos.

La estructura formal en una organización sirve para diseñar puestos de trabajo, especificar las labores a realizar en cada posición de trabajo y agrupar las tareas similares y/o relacionadas en departamentos, los que a su vez serán agrupados en unidades mayores que los contengan, de acuerdo a su especialización, similitud o vinculación de procesos y funciones. A este proceso se le denomina departamentalización, y refleja lo que Mintzberg denomina diseño de superestructura (tabla 1 ).

La departamentalización funcional lleva a que se definan secciones basándose en la división manufacturera del trabajo, con la expectativa de aprovechar la especialización de los empleados, para tener mayor productividad. En otras palabras, la departamentalización se apoya en el principio de división del trabajo, mediante el cual se espera que los empleados cooperen, dentro de sus áreas, con una parte del proceso y, por lo tanto, con los propósitos económicos de la empresa. En sentido moderno, la productividad:

Fuente: construcción propia.

Etimológicamente, diseño significa dibujar, marcar, delinear. Ver diccionario etimológico de Fernando Corripio Pérez (1973). 
"Es la situación mediante la cual se crea más de lo que se consume. Por lo tanto, el énfasis está en la creación de valor agregado, en vez de reducción de costo laboral. La productividad tiene entonces una dimensión social, no sólo económica, y debe ser entendida finalmente como sinónimo del desarrollo del recurso humano" (Prokopenlo, 1999).

Ahora bien, dependiendo del negocio en el que se mueva la empresa y del tamaño de ésta, esa unidad de trabajo recibe el nombre de área, división, sección, departamento, o simplemente, unidad, y por lo general se ubica espacialmente en un lugar específico dentro de la organización y recibe un nombre que la diferencia de las demás unidades.

En el diseño, la jerarquía permite establecer líneas formales de relación y de autoridad que van desde la cima hasta la base organizacional. Algunos autores consideran que las órdenes no pueden pasar por alto un escalón de la autoridad, ya que se corre el riesgo de debilitar esta autoridad.

Pareciera entonces que el diseño organizacional no puede prescindir de la jerarquía para lograr que las órdenes impartidas por los administradores se cumplan y para que las personas se muevan dentro de lo establecido, pues se cree que con la jerarquía se afirma la estabilidad del personal, y se establecen los límites de autoridad.

Por su parte, las normas y las reglas son una manera de regular y formalizar el comportamiento de los empleados dentro de una organización 7 . Para lograr el acatamiento de las reglas, algunos directivos se apoyan en el adoctrinamiento para sensibilizar a los empleados frente a la necesidad de obedecer tales normas. El adoctrinamiento:

"Se refiere a los programas y técnicas mediante los cuales las normas de los miembros de una organización son estandariza- das, para sensibilizarlos respecto a sus necesidades ideológicas" (Mintzberg, 2001, 376).

La estructura formal puede conducir a los empleados a que hagan algo porque la norma los obliga, o a que dejen de hacer porque la regla se los impi$\mathrm{de}$, porque deben ejecutar estrictamente las tareas asignadas por algún miembro de la dirección. Sobre este aspecto, Lipovetsky considera que:

"La gestión tayloriana del trabajo, preocupada por el problema de la haraganería y de las caídas de ritmo, se dedicó a transformar al obrero en un autómata sin pensamiento, ejecutante estricto de tareas fragmentarias preparadas por las oficinas de métodos (...) no hay más principio organizador que el cronómetro, la obediencia ciega, el salario basado en el trabajo a destajo" (Lipovetsky, 2002, 173).

$\mathrm{Al}$ parecer, lo que se busca es que el comportamiento, y quizás el pensamiento de los trabajadores, no se salga de lo establecido, ya que en una organización las normas expresan la pauta más indicada para determinadas actividades, con la intención de otorgar a tales actividades la mayor eficiencia y precisión posibles.

En consecuencia, el diseño organizacional acude a los denominados principios de la organización y a la división técnica del trabajo, el establecimiento de grupos formales, la definición de puestos, la determinación de reglas, funciones, comunicación formal y jerarquías, para estructurar la organización. La estructura de la organización puede también obrar recíprocamente con características del diseño de trabajo para influenciar los resultados del trabajador. Por ejemplo, dentro de una organización altamente centralizada donde los individuos tienen limitado control, el diseño de trabajo puede ayudar a reducir cualquier resultado potencialmente negativo de la centralización (Morgeson et al, 2010). Pero,

7 Se denomina regla a cualquier proposición prescriptiva. Es decir, aquel precepto o mandato que regula. Ver Nicola Abbagnano (1997). 
¿qué puede estar pasando en las relaciones humanas cuando se implementa el diseño organizacional y se afecta las interacciones entre los trabajadores?. ¿Tendrá algún impacto en el clima laboral??

A continuación se describirá brevemente la metodología desarrollada durante la investigación, para presentar posteriormente los hallazgos y conclusiones.

\section{Metodología}

Para Bonilla \& Rodríguez (2005) la investigación cualitativa es aquella en la cual el interés está puesto en captar la realidad social a través de los ojos de la población que está siendo estudiada. En la investigación que dio origen al presente artículo se emplearon la observación y la entrevista semiestructurada, como técnicas de recolección de información, mientras que el registro de los datos se realizó a través de notas de campo, grabaciones y fichas bibliográficas. Observar, con un sentido de indagación científica, implica focalizar la atención de manera intencional, sobre algunos segmentos de la realidad bajo estudio, tratando de captar sus elementos constitutivos y la manera cómo interactúan entre sí, con el fin de reconstruir inductivamente la dinámica de la situación (Bonilla \& Rodríguez, 2005, 228).

El estudio se realizó en tres empresas dedicadas a la producción y comercialización de empaques plásticos. Estas empresas contaban con entre 60 y 130 empleados, y sus directivos convinieron en que las compañías participaran en la investigación. Primero se desarrollaron las observaciones y luego las entrevistas. Ahora bien, es claro que no se pretende generalizar los hallazgos, asumiendo (como en el positivismo) que las demás empresas se comportan de igual manera. En contraste, se buscaba profundizar en el contexto organizacional para comprender la incidencia del diseño organizacional en los vínculos sociales y en la cooperación entre empleados de una misma compañía.
En las primeras observaciones, la presencia del investigador representaba un elemento extraño en el contexto de la empresa. Sin embargo, el observador fue ganando familiaridad, lo que facilitaba que los empleados se presentaran con mayor naturalidad en el trabajo. Durante la misma jornada de observación, se destinaba un momento para registrar la información obtenida, utilizando las notas de campo 9 . Dicho registro se elaboró a mano, tratando de que lo anotado fuera lo más "nativo" posible, es decir, escribiendo los términos tal cual habían sido escuchados, para evitar una inadecuada interpretación por parte del investigador.

Durante el trabajo de campo se realizaron seis visitas de observación, de cuatro horas cada una, en distintos días de la semana y a diferentes horas del día para captar variados momentos de la vida organizacional. Si bien no se efectuó una observación participante total, en el sentido de asumir los comportamientos de los trabajadores, si se desarrolló una observación participante en cierto grado, pues el investigador interactuaba con los empleados, compartiendo con ellos periodos y eventos laborales, para analizar los vínculos sociales y los actos concretos de colaboración y ayuda mutua entre los trabajadores. En la cafetería de la empresa, por ejemplo, pudo apreciarse la presencia de vínculos diferentes a los observados en la planta de producción, ya que los empleados dedican este momento y lugar para realizar intercambios verbales, bromear y establecer contacto físico, mientras planean algunas actividades que realizarán fuera de la empresa.

Adicionalmente, la observación se acompañó de entrevistas semiestructuradas. Galindo (1998, 281) define la entrevista como:

"Una conversación verbal entre dos o más seres humanos, cuya finalidad es lo que en verdad le otorga tal carácter. Es decir, en un sentido amplio, la entrevista es una conver-

8 El clima organizacional abarca las dimensiones del trabajo, de la dirección, las características del grupo de trabajo, y de la organización. Jones y James describieron características de trabajo por el grado de autonomía, de variedad, de desafío, de importancia, presión, y de ambigüedad/claridad y conflicto (Larsson et al, 2008).

9 Las notas de campo son descripciones de los procesos sociales en los contextos donde suceden y anotaciones reflexivas sobre la aplicación de estrategias y técnicas de investigación (Galeano, 2004, 48). 
sación que establecen un interrogador y un interrogado para un propósito expreso".

La siguiente fase consistió, entonces, en con Ilustraciónr la muestra de participantes para combinar y contrastar información proveniente de observaciones, de conversaciones informales y de entrevistas con los empleados.

Para Galeano, las razones de selección de los informantes son de comprensión y de pertinencia y no de representatividad estadística (Galeano, 2004, 34). Por consiguiente, se consideraron distintas razones para la selección de la muestra: se entrevistaron personas de diferentes áreas de la organización, empleados de la parte administrativa y de la parte operativa, trabajadores de poca y mucha antigüedad en la empresa, hombres y mujeres. Se entrevistó un grupo preliminar de nueve personas, de acuerdo a la información que poseían y la disponibilidad manifestada durante las observaciones. Posteriormente, se fue graficando un nuevo grupo, conformado por ocho personas más, de acuerdo a la información suministrada por los empleados del primer grupo. Esto se hizo con el fin de poder profundizar en el fenómeno estudiado. Las entrevistas se desarrollaron siguiendo el principio de saturación ${ }^{10}$.

A partir de lo planteado en el marco teórico de la investigación, se inició la lectura de la información recolectada para con graficar las categorías de análisis que permitieran interpretar los hallazgos del estudio ${ }^{11}$. En particular, departamentalización, puestos de trabajo, jerarquía y reglas y normas son los aspectos del diseño organizacional que emergieron de la información recolectada en el trabajo de campo y que serán abordados a continuación. Es necesario aclarar que por la misma naturaleza de la investigación, ésta no tiene pretensión de generalizar los resultados, sino comprender el fenómeno social-organizacional en sí mismo.

\section{Algunos parámetros de diseño organizacional}

Es importante precisar que no todos los elementos o parámetros del diseño organizacional planteados por Mintzberg aparecieron en las entrevistas. Veamos los aspectos emergentes en la siguiente tabla, antes de abordarlos separadamente.

Tabla 2. Características del diseño organizacional que emergieron en la investigación ${ }^{12}$

\section{Parámetros del diseño organizacional}

El diseño organizacional, como categoría de análisis que emergió de la información en la investigación, se caracteriza por:

Establecer una departamentalización funcional de acuerdo con la división manufacturera del trabajo, para buscar la especialización de los empleados en la organización.

La definición del puesto de trabajo de acuerdo con la máxima el hombre adecuado en el lugar apropiado y apoyado por la rotación de personal para la conservación de dicha máxima en beneficio de la productividad.

El establecimiento de distintos niveles jerárquicos para el cumplimiento de la unidad de mando y aseguramiento de la autoridad, que contribuyan con el buen funcionamiento de toda la organización.

La declaración de reglas, normas, directrices tales como el manual de funciones y el reglamento interno de trabajo que aseguren la adhesión de los empleados con los objetivos económicos de la compañía.

10 Es necesario precisar que en las entrevistas semiestructuradas, el investigador se aproxima al objeto de estudio con algunas preguntas derivadas del marco teórico, las hipótesis y los objetivos de investigación; y a partir de las respuestas dadas por los participantes, van emergiendo nuevas preguntas. Las preguntas realizadas indagaban por los actos de cooperación y apoyo entre los empleados, así como por aquellos aspectos que los facilitaban e impedían. También se preguntó por el tipo de vínculos que se construían con compañeros de trabajo y por los aspectos que limitaban o favorecían dichos vínculos. Otro grupo de preguntas apuntaba a la relación entre el grado de cooperación presente con compañeros de trabajo y los vínculos existentes entre los mismos.

11 Para Galeano (2004), las categorías son ordenadores epistemológicos, campos de agrupación temática, supuestos implícitos en el problema y recursos analíticos. Como unidades significativas dan sentido a los datos y permiten reducirlos, compararlos y relacionarlos.

12 Fuente: construcción propia. 


\subsection{La departamentalización funcional}

Además de la departamentalización funcional, existen otro tipo de departamentalizaciones que no fueron objeto de estudio en la investigación. Solamente se abordó aquella que implicaba una unidad clara y estrictamente diferenciada de las demás a partir de la división técnica del trabajo. Otros conceptos afines a departamentalización funcional son grupos formales, comités, equipos de trabajo, etc.

Con la división de labores y la separación de las personas en unidades se disminuye la posibilidad de interactuar con los compañeros, y recuérdese que la interacción facilita que los sujetos se vinculen a través de una solidaridad mecánica, la cual propicia el surgimiento de una cooperación especial que contribuye con la cohesión social de todo colectivo humano: la cooperación espontánea.

A continuación, entonces, se revisarán las expresiones de algunos entrevistados referidas al efecto que la separación entre unidades tiene sobre la cooperación entre los empleados. El hecho de que las personas se encuentren en departamentos diferentes, separados físicamente, minimiza las interacciones entre los trabajadores y circunscribe su actuación a su propio departamento. Al preguntarle a uno de los entrevistados sobre las relaciones que se establecen con personas de otras áreas, éste comenta:

"Los otros no tienen nada que ver. Es decir, si en sellado se dañó una máquina, en extrusión nada que ver. Lo que pasa es que el jefe de sellado es el mismo de extrusión, entonces las relaciones entre secciones se conectan más por los jefes. Pero no por los operarios. Cada uno está en su sección, haciendo su trabajo, y si en sellado se dañó una máquina, entre ellos allá ven cómo lo solucionan, porque son secciones totalmente apartes" (Ent. 3).

Puede observarse que la departamentalización distancia a las personas porque sus actividades y tareas no se conectan ni relacionan entre sí. Incluso los mismos empleados consideran que los compañeros de otras secciones no tienen nada que ver con su área de desempeño y que, por consiguiente, las relaciones con ellos son muy pocas, apreciándose así un efecto de la departamentalización sobre la interacción entre los trabajadores. Al respecto, el mismo entrevistado agrega que:

“¿Usted me ayuda cuando a mí se me daña algo sabiendo que usted es de sellado y yo de impresión? no! Si [los empleados] se conocen es porque trabajan todos acá, pero no porque los trabajos interfieran" (Ent. 3).

Si consideramos que Goffman define la interacción como:

"la influencia recíproca de un individuo sobre las acciones del otro cuando se encuentran ambos en presencia física inmediata" (Goffman, 2001, 27).

Puede pensarse que la departamentalización afecta la cooperación entre los empleados debido a que limita la posibilidad de que éstos interactúen unos con otros, dada la separación física de los empleados y la delimitación de tareas.

Es importante resaltar que en la empresa los actores:

"Realizan el mecanismo básico de interacción organizacional a través del lenguaje; para generarse, mantener sus límites y garantizar estabilidad interna, las organizaciones utilizan la comunicación" (Echavarría, 2000, 75).

De igual forma, y dado que la división del trabajo busca la especialización para que el trabajador sea más eficiente y la empresa más rentable, la departamentalización funcional afecta la cooperación entre los trabajadores cuando alguno de ellos no puede colaborarle a su compañero porque no conoce la tarea de éste. Al preguntar a un empleado sobre la ayuda que prestaría a otro trabajador que se encuentra en una sección diferente, señala:

"Por ejemplo, en este momento hay máquinas muy nuevas. Yo casi no las sé uti- 
lizar. Muchas veces van y me preguntan algo de esa máquina, y no sé, porque como no las he trabajado" (Ent. 8).

Además del efecto sobre la colaboración por no conocer el trabajo del otro, el alejamiento físico determinado por la departamentalización genera un aislamiento entre los miembros de estas unidades. En la expresión del siguiente entrevistado puede observarse la consecuencia del distanciamiento físico de los trabajadores sobre la interacción y la cohesión del grupo de trabajo:

"Nosotros, aquí en la oficina, parecemos como aparte de la fábrica. Parecemos como si viviéramos en un mundo aparte de muchas cosas que pasan allá, parecemos como aislados" (Ent. 4).

La separación física disminuye la posibilidad de que los empleados se relacionen y comuniquen entre sí, logrando que las personas se perciban así mismas "como aisladas" unas de otras, de acuerdo con el área o sección a la que pertenezcan dentro de la empresa. Sobre ello, el mismo empleado complementa:

"Ellos muchas veces ni siquiera saben cómo se llama un trabajador. O los confunden, y lo que yo les decía ahorita: Acá nos celebramos los cumpleaños. ¡Nosotras! Nosotras tenemos una alcancía, guardamos plata, compramos una tortica o hacemos cualquier cosa" (Ent. 4).

Cuando la entrevistada enfatiza y reitera "nosotras" quiere dejar claro que la celebración del cumpleaños es una actividad de y para los de su sección únicamente, y que en dicha actividad no se incluye a otros empleados de áreas diferentes, porque con aquellos otros no existe la misma cohesión que se tiene con los de su sección, por la minimización de los intercambios verbales y la poca interacción existente entre ellos.

En contraste, por la misma razón por la cual se afecta la cooperación entre personas de distintos departamentos, la cooperación entre personas del mismo departamento se ve favorecida, por el con- tacto social que esto supone. Es evidente que la cercanía física permite a los empleados interactuar entre sí, comunicarse y generar lazos sociales entre ellos más fuertes que los que podrían desarrollarse con aquellas personas a las cuales casi no se ve y $\mathrm{ni}$ siquiera se les conoce el nombre.

Precisamente la posibilidad de relacionarse con los demás facilita que se construya (entre los empleados) vinculaciones distintas a las relaciones establecidas por el organigrama, producto de la presencia de sentimientos comunes entre las personas, pues como señala Durkheim:

"Para que una cosa sea objeto de sentimientos comunes, la primera condición es que sea común, es decir, que esté presente en todas las conciencias y que todas puedan representársela desde un solo e idéntico punto de vista" (Durkheim, 1967, 111).

Así entonces la departamentalización se ocupa sólo de los aspectos económicos y se olvida de los aspectos más humanos de la organización. Se olvida que los empleados son seres sociales por naturaleza, que necesitan de la interacción y de la posibilidad de hablar y comunicarse, para sentirse reconocidos por los otros.

\subsection{El puesto de trabajo}

En esta categoría se puede hablar de ocupación, puesto, cargo, posición, etc. En la investigación se prefirió mantener el término "puesto de trabajo" para utilizar la misma expresión a la que se referían los distintos informantes o participantes en el estudio. La particularidad de esta categoría está dada por el diseño y la descripción del cargo, la cual delimita tajantemente cuáles son las funciones de dicho cargo y en las que se debe concentrar el empleado.

El puesto de trabajo establece un límite a la acción de las personas. Expresiones como "limítese a hacer su trabajo", "esto no es función suya", "es que yo no puedo hacer eso" son expresiones que reflejan la forma como el puesto de trabajo restringe a la persona y su comportamiento. En consecuencia, si 
el empleado está pensando que fue contratado para hacer un trabajo específico y que su desempeño se evaluará en función de éste, no hará otra cosa si cree que pone en riesgo su tarea, y por consiguiente, su permanencia en la empresa. Al preguntarle a Marta sobre su puesto de trabajo, menciona:

"Si, cada una se concentra en lo suyo. Si, en el trabajo".

Y sobre la colaboración brindada a los compañeros en momentos de discrepancia, otra empleada señala:

"Hay cosas en las que definitivamente yo no puedo actuar, ¿cierto? Entonces María o Carlos lo harán. Pero depende mucho de las dificultades que tengan ellos y hasta dónde me dejan llegar" (Ent. 1).

Dado que el puesto de trabajo le indica al trabajador a lo que se debe remitir, puede suceder, como en el caso anterior, que un empleado decida no involucrarse con otros porque el mandato empresarial le indica que se debe concentrar en lo suyo y no inmiscuirse en las funciones del otro. Y los jefes no se lo permiten porque en la relación jefe-subordinado se aprecia una relación de poder en la que el subordinado es sometido a la voluntad del patrón. En este sentido, Foucault sostiene que:

"En las relaciones humanas el poder está siempre presente: me refiero a cualquier tipo de relación en la que uno intenta dirigir la conducta del otro (...) y es preciso subrayar que no pueden existir relaciones de poder más que en la medida en que los sujetos son libres. Si uno de los dos estuviese completamente a disposición del otro y se convirtiese en una cosa suya, en un objeto sobre el que se puede ejercer una violencia infinita e ilimitada, no existirían relaciones de poder (Foucault, 1994, 126).
La idea de autonomía reside, de acuerdo con Foucault, en la existencia de sujetos que cuestionen sus propias leyes, pero muy probablemente los trabajadores no pueden cuestionar las normas y reglamentos de la organización por temor a perder su empleo.

La formalización del comportamiento es un parámetro mediante el cual el diseño restringe las prácticas de libertad de los empleados, induciéndolos a que hagan exclusivamente lo que el proceso de trabajo requiere, puesto que dicha formalización es usada para asegurar la consistencia mecánica que conduce a la producción eficiente (Mintzberg, 2001). Esta restricción al trabajador se observa cuando a una empleada se le pregunta por quién tiene la responsabilidad de realizar el grupo primario $^{13}$, y ella responde:

"Esa es su tarea y ellos se tienen que enterar que esa es su función. El manejo del grupo primario es responsabilidad de ellos. No es la mía. La mía si mucho será dar ideas para que solucionemos algo, no más" (Ent. 1).

Por consiguiente, la delimitación de las tareas está haciendo que los empleados se concentren exclusivamente en su labor y olviden que sus compañeros necesitan de su colaboración y de su apoyo. Al consultarle a otro participante sobre la posibilidad de ayudarle a un compañero que tenga una dificultad en el desempeño de su función, cuando se sabe que esta labor corresponde a los auxiliares, responde:

"Son ellos los que nos tienen que ayudar a nosotras. Nosotras no podemos estar con la otra compañera, tiene que ser por la última. Pero de resto, ellos son los que están pendientes de nosotras.

Y ¿por qué no pueden estar con la otra compañera?

No, no es permitido" (Ent. 9).

13 En algunas organizaciones, los empleados se estructuran en los denominados grupos primarios para efectos de desarrollar una mejor y más directa comunicación sobre los aspectos que atañen al buen funcionamiento de sus áreas de trabajo. 
No es permitido. ¿No es acaso esta expresión la manifestación de una limitante a la acción de las personas? Una limitante que se precisa mediante el diseño de posiciones descrito en la tabla 1 y que se concreta con la formalización del comportamiento, con la capacitación y con la estandarización del contenido de la tarea explicitada en los manuales de funciones.

De otro lado, la literatura administrativa considera un error de la organización atribuir responsabilidad a quien carece de autoridad. Así, puede apreciarse los limitantes a la acción de las personas, cuando se argumenta que:

"Los subordinados tienden a creer que un puesto lo permite todo, y a olvidar por tanto que su autoridad se limita al departamento al que pertenecen y que debe ser acorde con las pautas de control" (Koontz \& Weihrich, 1998, 373).

La consecuencia de esto es que los trabajadores se sienten impedidos para ayudar a sus compañeros en los problemas de estos últimos, porque deben ceñirse a sus funciones, aunque esto minimice el contacto social con los compañeros. Con el anterior mandamiento de Koontz \& Weihrich se limita la cooperación aun sabiendo que un trabajador tiene la capacidad para colaborar en la solución de un problema.

La delimitación del puesto de trabajo alcanza tal punto que propicia un total desinterés por el trabajo del otro, llagando incluso hasta una posible insolidaridad e individualismo ${ }^{14}$.

Parece evidente que para algunos directivos es más importante la eficiencia técnica de los empleados que los intereses de éstos y las relaciones humanas, en el puesto de trabajo. El clima organizacional constituye una condición importante para el trabajo diario. Un clima favorable es caracterizado por las descripciones de las funciones claramente indicadas, y una situación de trabajo que permita que el individuo tenga influencia sobre su trabajo. Además, un clima favorable implica que el individuo puede realizar su trabajo dentro de un ambiente de apoyo donde tenga un sentido de comunidad con sus compañeros y donde se reconocen las habilidades del individuo (Larsson et al, 2008).

Pero en la perspectiva "racional" del diseño, prima el interés por la rentabilidad sobre la preocupación por ambientes sanos de trabajo, donde las personas puedan desarrollar sus habilidades sociales, generándose un clima organizacional adecuado y un nivel alto de productividad, por la satisfacción laboral que esto supone. Sobre la destreza necesaria para desempeñar una labor, un entrevistado explica por qué se ubicó a una empleada en determinado cargo, y expresa: [La empleada asignada al cargo] se preguntaba:

“¿Pero a mí por qué en esta máquina y las otras en las más modernas?. Muchas veces es porque se le ve las condiciones a ella. ¿Porqué a la otra la ponen en las mejores máquinas?; entonces las otras también empiezan a decirle indirectas y eso se vuelve en un problema" (Ent. 6).

La preponderancia de lo técnico sobre lo social para alcanzar los objetivos económicos de la empresa genera cierta resistencia en los trabajadores $y$, a su vez, altera la cohesión social del grupo, por los efectos que sobre el clima laboral se tienen. Tal como lo manifiesta la cita anterior, algunas medidas llevan a que unos empleados no se sientan contentos con la decisión de ubicar a ciertos empleados en las mejores máquinas, y descarguen su impulso sobre los compañeros lanzándole indirectas, teniéndose como consecuencia un efecto posterior sobre la cooperación espontánea entre ellos.

14 Algunos autores diferencian del contenido del puesto y el contexto del puesto. El contenido se relaciona con el trabajo en si. Incluye conceptos como la idea de logro y de reconocimiento o crecimiento personal. Los factores del contenido del puesto se consideran intrínsecos. El contexto se refiere a las políticas de la organización, incluidos los aspectos técnicos de supervisión, el ambiente de trabajo y las interrelaciones con los demás empleados, todos estos denominados aspectos extrínsecos (Terry, 1984). 
Adicionalmente, cuando una persona es trasladada de un puesto de trabajo a otro, ese trabajador necesita empezar un proceso de adaptación al cargo $y$ de encuadre social con sus nuevos compañeros. Como en el caso de Cristina cuando afirma que:

"Yo estuve en la recepción dos años. Para mí fue muy duro el cambio de la recepción a nómina. Me vi con más responsabilidad. Este cargo para mí es muy importante, muy delicado. Aparte de eso, yo iba mucho a la planta. Y recién empecé en este puesto, me dijeron que a mí se me estaba "subiendo el puesto a la cabeza", que porque ya no iba a la planta, que ya no saludaba."

En el caso anterior, se pueden apreciar efectos que los cambios de puestos tienen sobre las personas. En primer lugar, el empleado debe adaptarse a un nuevo jefe, nuevos compañeros y nuevas funciones. Además, en el nuevo cargo se necesitan otras habilidades o competencias, para lo cual las personas tienen que entrar a capacitarse, y en ocasiones dejar de realizar ciertos actos que hacen parte de sus costumbres. Igualmente, estos cambios implican un distanciamiento con los antiguos compañeros de trabajo, poniéndose en riesgo la interacción y la vinculación con éstos.

\subsection{La Jerarquía}

Independientemente de la posición que ocupan en la empresa, los trabajadores entrevistados hacen referencia las relaciones jefes-subordinados, a la presencia de autoridad, a las directrices emanadas de la dirección y a los flujos ascendentes y descendentes de la comunicación. La categoría jerarquía permite agrupar las expresiones de los participantes y, además, las otras categorías como poder y autoridad no fueron objeto de estudio. La jerarquía no sólo busca garantizar la unidad de mando y la obediencia, sino que propende porque las personas no se salten instancias y se conserve lo formal y lo establecido en la organización. Sobre ello, Patricia sostiene:

"Muchas veces yo me siento muy impotente. Porque yo no tengo el poder en mu- chas ocasiones para actuar y apoyar a los compañeros. Lo máximo que puedo hacer es hablar con Karla, y muchas veces hablarlo directamente con Antonio" (Ent. 4).

Cuando la empleada afirma "yo no tengo el poder en muchas ocasiones" se refiere a que su autoridad dentro de la jerarquía organizacional le establece un límite. $\mathrm{Y}$ al conocer ese límite ella se siente impotente. Cuando Patricia se refiere a Karla y a Antonio sabe que, por el poder formal que la jerarquía les otorga (pues ocupan cargos de mayor nivel jerárquico), ellos pueden ayudar a sus compañeros de trabajo, ya que su propio cargo no se lo posibilita. De la misma manera, con la jerarquía se puede crear un distanciamiento entre los distintos niveles organizacionales, pues:

"No es nuevo que los burócratas, especialmente en sus grados superiores, constituyan una clase social, con intereses propios, distintos y contrastantes respecto de los de otras clases" (Mouzelis, 1991, 85).

Y esta diferencia de niveles crea un distanciamiento de la relación entre los miembros de una organización, como lo relata una entrevistada que fue ascendida a otro nivel jerárquico, considerando que el principal efecto de este cambio se dio en la relación con sus compañeros de trabajo:

"Yo interactuaba mucho con los compañeros de mi área. [Y después] las cosas cambiaron. Vi que tenía más responsabilidades. Tanto así que ellos me dijeron que yo había cambiado mucho. Ellos al principio no entendían eso, entonces eso fue muy difícil. [Además] yo interactuaba mucho con las administradoras de los almacenes, y ya casi no. Perdí mucho el contacto con ciertas personas, por el cambio de puesto" (Ent. 4).

Estos ascensos pueden generar efectos sobre la cooperación espontánea, dadas las prohibiciones y las obligaciones que se le exige a algunos cargos. Ya no se trata de la obligación moral de trabajar por la satisfacción de hacerlo, sino del deseo personal de 
triunfar en las nuevas funciones, de sentirse responsable de la propia tarea, de progresar. Al respecto Aubert \& Gaulejac consideran que:

"La estructura jerárquica exige una sumisión y una obediencia constantes y el respeto de las prohibiciones: hay que obedecer las órdenes, mostrarse dócil, respetar los horarios y sobre todo plegarse a la jerarquía" (Aubert \& Gaulejac, 1993: 133).

Pero tal sometimiento y obediencia a la jerarquía trae consecuencias para la cohesión social y la relación entre dos empleados, cuando uno de ellos es trasladado a un cargo superior. "Perdí mucho contacto con ciertas personas", afirmó la entrevistada, reflejando el efecto que tal decisión administrativa tiene sobre la interacción social. Ahora bien, considerar estos efectos no es, en palabras de Calderón:

"Una mirada ingenua al ser humano, es considerarlo en su integralidad sin descontextualizarlo de una realidad en la cual vive: actúa y decide; no es simplemente un instrumento de producción, es una persona con sentimientos, valores, intereses, imaginación, palabras y pensamientos" (Calderon, 2004, 75).

\subsection{Las Normas y las Reglas}

Los reglamentos internos de trabajo, los manuales de convivencia, así como los memorandos son mecanismos a través de los cuales se hacen explícitas las normas y las reglas en las empresas estudiadas. En general, el discurso de los trabajadores hace referencia reiterada (sin mencionar necesariamente la forma) a todas esas maneras de regular y formalizar el comportamiento humano al interior de la empresa. Es una sujeción a la norma lo que está afectando la cooperación espontánea, pues como dice Mouzelis:

"Las normas, los procedimientos, la oficina son a menudo objeto de una especie de santificación por parte de los funcionarios; incluso sus actividades más técnicas tien- den así a ser intocables" (Merton, citado por Mouzelis, 1991, 85).

En consecuencia, las personas no querrán salirse de la norma por temor a perder el empleo, como lo sugiere un empleado, cuando expresa:

"Si algún día montara una empresa, las reglas serían esas. Y el que no las cumpla, para afuera. $\mathrm{Si}$, porque empiezan a hacer un ambiente pesado, y no hay cosa más horrible que uno trabajar incómodo" (Ent. 2).

Tal sometimiento es un reflejo de la dinámica organizacional de algunas empresas colombianas que, como menciona Uribe (2004), se amparan en el discurso de la transformación cultural, diseñan y ejecutan sofisticados y sutiles mecanismos de sometimiento los cuales se convierten en verdaderos dispositivos de poder. Para Uribe, estos dispositivos son trazados:

"Para conseguir del trabajador la voluntad, y un cuerpo dócil que se adhiera a las exigencias del trabajo y a los intereses de la organización; dispositivos y mecanismos que son asumidos por administradores $y$ trabajadores como verdades y rutinas que no se interrogan" (Uribe, 2004).

Sobre la norma, un entrevistado manifiesta lo siguiente:

"Yo he sido víctima de muchas cosas perversas. Porque aquí obligan a la gente a ser callada, a no hablar con nadie. Haga su trabajo usted solo y no mire para los lados" (Ent. 7).

Mediante las normas, el empleado en la organización es un embrague de toda la maquinaria, interesada en los beneficios económicos. Sobre ello, Weber considera que el empleado:

"Está encadenado a su actividad a través de toda su existencia material y espiritual. Por lo general, es un simple engranaje de un mecanismo siempre en marcha que le 
ordena ir en un sentido esencialmente fijo. El funcionario debe realizar tareas especializadas y, normalmente, no puede arrancar ni detener el mecanismo" (Weber, 2001, 83).

Cuando se interroga a un informante por la ayuda que podrían prestarse unos empleados a otros ante un problema específico, menciona:

"El auxiliar es el que les debe ayudar a montar el rollo, él es el que debe ayudar. ¿Debe, cómo así? Debe. Es su trabajo. Para eso está. Es su labor, ¿cierto? El es el que debe ayudar. Si a las máquinas se les dañan las bandas, él se las debe venir a colocar" (Ent. 2).

Cuando el entrevistado dice "debe" es claro que se refiere a que ese es el trabajo del auxiliar y de nadie más, porque así lo recita el manual de funciones y, además, porque el reglamento está diseñado para que los trabajadores cumplan las funciones que les son encomendadas. Con el reglamento interno de trabajo y demás normas implícitas y explícitas en la organización, se definen dispositivos para ejercer control sobre los actos de los empleados, los cuales incluyen la interacción y el diálogo cara a cara. Con las normas, como mecanismo que busca regular el comportamiento de los empleados, se afecta la cooperación espontánea entre éstos. Al respecto, el siguiente señalamiento de Foucault permite ampliar la perspectiva de norma que se viene desarrollando:

"A partir de los siglos XVI y XVII, en el ejército, en las escuelas, los hospitales, los talleres y otros espacios, se desplegaron toda una serie de técnicas de vigilancia y de control, de mecanismos de identificación de los individuos, de cuadriculación de sus gestos y de su actividad, que fueron conformando determinados tipos de productores. El capitalismo moderno necesita para su desarrollo capitalistas, es decir, sujetos que actúan de acuerdo a un determinado ethos, impregnados de una determinada mentalidad empresarial" (Foucault, 1994, 27).
Pero cuando esas normas son incorporadas condicionan la forma de comportamiento de los empleados, quizás sin un análisis previo por aparte de éstos o sin el deseo de hacerlo, porque se hace por la necesidad de laborar, y no por la convicción de que esas reglas sean convenientes para su comportamiento o estilo de vida.

"Uno de los hallazgos más consistentes en la literatura de organizaciones es que las mediciones afectan el comportamiento. La mayoría de las personas tratarán de tener éxito en las mediciones, incluso si no hay consecuencias directas e inmediatas" (Pfeffer, 1996).

\section{Conclusiones}

Los trabajadores siguen siendo personas después de unirse a laborar en una empresa, y por lo tanto, también tienen sus intereses, objetivos y expectativas. Algunas de esas expectativas van más allá de lo económico: construir relaciones y vínculos con otras personas, comunicarse, interactuar y generar cohesión y cooperación, son igualmente importantes para los empleados.

La departamentalización funcional, derivada de la división del trabajo, conduce a una mayor eficiencia en la empresa. Adicionalmente, los empleados que pertenecen al mismo departamento cooperan más fácilmente entre ellos dados los vínculos que han desarrollado, como consecuencia de la interacción social a la que estaban expuestos.

Sin embargo, dada la separación de tareas entre diferentes divisiones y la poca o casi nula interacción entre empleados de diferentes áreas, la cooperación y los vínculos sociales entre éstos últimos es muy limitada. La estricta delimitación de las tareas asignadas a cada puesto de trabajo, sumadas al hecho de que, en ocasiones, a los empleados se les prohíbe inmiscuirse en las tareas de los compañeros, limita la interacción, los intercambios sociales y la cooperación entre los empleados. 
Se sabe que las estructuras burocráticas son un obstáculo al diálogo (Chanlat \& Bedard, 1999), y además, la jerarquía genera relaciones distantes entre jefes y empleados, porque los primeros tienen temor a perder su autoridad y porque hacen que los segundos no se tomen atribuciones que no les "corresponden". Esas distancias observadas entre un nivel y otro (jefes y empleados) no solo limitan la interacción social sino que, incluso, generan distanciamientos en los casos donde ya existían vínculos sociales entre dos trabajadores.

El estricto sometimiento a las reglas y normas hace que algunos empleados se sientan cohibidos para cooperar abiertamente con compañeros (y de construir relaciones sociales con ellos) porque temen ser amonestados. En esos casos, es más importante el cumplimiento de la regla que el mejoramiento de la productividad, a través de la cooperación. Ahora bien, dado el funcionalismo inherente a la práctica administrativa, se requieren ciertas disposiciones por parte de directivos, encaminadas al logro de los objetivos de la empresa. Y las medidas que implica el diseño organizacional contribuyen con la productividad de la empresa. Sin embargo, se requiere que los administradores y directivos tengan en cuenta que también es su responsabilidad con los empleados minimizar los efectos del diseño sobre aspectos sociales tales como la vinculación, la cooperación, la interacción y la cohesión social. Lo ideal es que con la productividad no sólo gane la empresa sino también los trabajadores, y que en el interés de diseñar la organización se contribuya con la construcción de un ambiente sano de trabajo, porque cuando una persona se siente satisfecha con su trabajo, se sentirá más impulsada para desarrollar con mayor desempeño sus tareas.

No se pretende entonces volver atrás en las medidas administrativas que han demostrado eficiencia. No se busca desacreditar los logros que permite la división técnica del trabajo. No se trata de eliminar las jerarquías cuando se sabe que favorecen la eficacia. No se intenta crear organizaciones sin normas ni reglamentos o empresas donde no exista una clara definición y diseño del puesto de trabajo. Se trata, en primera instancia, de reconocer los impactos que estas medidas y parámetros del diseño organizacional tienen en otras esferas organizacionales, y en segundo lugar, de propiciar espacios de reflexión para que los diseñadores, quienes dicen que se preocupan por el factor humano en la organización, aboguen por medidas y decisiones administrativas que contrarresten o aminoren los impactos de esos parámetros de diseño en las compañías.

Por su definición funcional, la organización necesita de la cooperación de todos sus miembros, pero algunos administradores se están perdiendo de un tipo de cooperación especial. De esa cooperación espontánea que emerge naturalmente en la organización producto de las vinculaciones entre los empleados. Y se están perdiendo de ella porque algunos parámetros del diseño limitan las interacciones entre los empleados. En esta perspectiva, la interacción social también ha sido aclamada por investigadores como Chena y Huang, quienes afirman que los directivos deben ser conscientes de la importancia de la interacción social en el acoplamiento del clima de organización y de la estructura organizacional. Es imprescindible que las firmas desarrollen la interacción social entre sus trabajadores y den las bases para compartir. Además, las firmas deben diseñar cuidadosamente contextos de organización apropiados para facilitar la interacción social (Chena \& Huang, 2007).

\section{Referencias}

Abbagnano, N. (1997). Diccionario de filosofía. México: Fondo de cultura económica.

Aktouf, O. (2001). La administración. Entre la tradición y la renovación. Cali: Universidad del Valle.

Aubert, N. \& Gaulejac, V. (1993). El coste de la excelencia. Barcelona: Paidós.

Barnard, C.I. (1971). The functions of the executive. Harvard University.

Barrera, E, Rodríguez, J. Bedoya, J. Una perspectiva analítica para transformar los procesos de institucionalización organizacional. En: Reflexión política, (2008), No 19. IEP-UNAB: 148-16.

Beckman, S. L. (2009). Introduction to a Symposium on Organizational Design. California management review, Vol. 51, No. 4

Bendix, R. (1974). La burocracia. En: D.L. Sills (Eds), Enciclopedia Internacional de las Ciencias Sociales. Madrid: Aguilar.

Bonilla, E. \& Rodriguez, (1997). Más allá del dilema de los métodos. Bogotá: Norma. 
Calderón, G. (2004). Lo estratégico y lo humano en la dirección de las personas. Pensamiento \& gestión, 16. Universidad del Norte: $158-176$

Chanlat, A. \& Bédard, R. (1990). L'individue dans L'organisation, les dimensions oubliés. Québec: Pul-Eska. Traducción libre de Rodrigo Muñoz.

Chena, C. \& Huang, J. (2007). How organizational climate and structure affect knowledge management-The social interaction perspective. International Journal of Information Management. Vol. 27: 104-118

Corripio, F. (1973). Diccionario Etimológico General de la lengua Castellana. Ed. Bruguera, España.

Echavarría, R. (2000): La empresa emergente: la confianza y los desafíos de la transformación, Buenos Aires: Granica.

Foucault, M. (1994). Hermenéutica del sujeto. Madrid: La Piqueta.

Freud, S. (1981). En: Obras completas, Madrid: Biblioteca Nueva. Vol. 3.

Galeano M. E. (2004). Diseño de proyectos en la investigación cualitativa. Medellín: Universidad Eafit.

Galindo, J. (1998). Técnicas de investigación en sociedad, cultura y comunicación. México: Addison Wesley.

Giddens, A. (1998). El capitalismo y la moderna teoría social: un análisis de los escritos de Marx, Durkheim y Weber. España: Idea books.

Goffman, E. (2001). La presentación de la persona en la vida cotidiana. Buenos aires: Amorrortu.

Goffman, E. (1991). Los momentos y sus hombres. Madrid: Paidós.

Goodin, R.E. (1996). The theory of administración pública?. IX Congreso Institutional Design. Cambridge University Internacional del CLAD sobre la reforma del Press, Cambridge.

Hodge, B. \& otros. (1998). Teoría de la organización. Un enfoque estratégico. Madrid: Prentice Hall.

Koontz, H. \& Weihrich, H. (1998). Administración, una perspectiva global. México: McGraw Hill.
Larsson, S, Pousette, A. \& Torner, M. (2008). Psychological climate and safety in the construction industry-mediated influence on safety behavior. Safety Science Vol. 46: 405-412.

Lipovetsky, G. (2002). El crepúsculo del deber. La ética indolora de los nuevos tiempos democráticos. Barcelona: Anagrama.

Mintzberg, H. \& Quinn, J.A. (1993). El proceso estratégico: conceptos, contextos y casos. México: Prentice Hall.

Mintzberg, H. \& Quinn, J.A. (2001). Diseño de organizaciones eficientes. Buenos Aires: El Ateneo.

Morgeson, F. Dierdorff, E. \& Hmurovic, J. (2010). Work design in situ: Understanding the role of occupational and organizational context. Journal of Organizational Behavior. Vol. 31 351-360.

Mouzelis, N. (1991). Organización y burocracia. Un análisis de las teorías modernas sobre organizaciones sociales. Madrid: Península.

Nisbet, R. (1974). Cooperación. En: D. L. Sills. (Eds), Enciclopedia internacional de las ciencias sociales. Madrid: Aguilar.

Pfeffer, J. (1996): Ventaja competitiva a través de la gente, México: CECSA

Prokopenlo, J. (1999): Productivity promotion Organizations. Evolutions and experience. Organización Internacional del Trabajo.

Visscher, K. \& Visscher-Voerman, J. I. (2010). Organizational design approaches in management consulting. Management. Decision Emerald Group Publishing Limited. Vol. 48 No. 5: 713-731.

Weber, M. (2001). ¿Qué es la burocracia?. México: Coyoacán.

Wilkens, R. \& London, M. (2006). Relationships between climate, process, and performance in continuous quality improvement groups. Journal of Vocational Behavior. Vol. 69 510-523

Zapata, A. (2008). Análisis y Diseño Organizacional: De la estructura funcional a la organización vacía. Cali: Universidad Del Valle. 